\title{
Data Enhancement for Active Thermography
}

by S. Götschel*, M. Weiser*, Ch. Maierhofer** and R. Richter**

*Zuse Institute Berlin, Berlin, Germany, \{goetschel, weiser\}@zib.de

**BAM Federal Institute for Materials Research and Testing, Berlin, Germany,

\{christiane.maierhofer, regina.richter\}@bam.de

\section{Abstract}

Pulse thermography is a non-destructive testing method based on infrared imaging of transient thermal patterns. Heating the surface of the structure under test for a short period of time generates a non-stationary temperature distribution and thus a thermal contrast between the defect and the sound material. Due to measurement noise, preprocessing of the experimental data is necessary, before reconstruction algorithms can be applied. We propose a decomposition of the measured temperature into Green's function solutions to eliminate noise.

\section{Introduction}

Image enhancement and noise reduction for IR-data is an important step for the quantitative reconstruction of defect depth and size. Most common is the application of standard tools from image processing, like Gauss- and Medianfilters (in space), or the moving average filter (in time) [1]. Several other data filtering techniques, e.g., the Wiener filter, are discussed in [2]. In [3] a spatio-temporal filtering based on averaging over sub-blocks is proposed. The size of the sub-blocks is increased with increasing time, leading to both compression and improved signal-to-noise ratio.

An algorithm based on an analytic solution of the heat conduction equation for the enhancement and analysis of thermographic images was proposed in [4] for the localization of heat sources.

We follow an approach that is similar to the Thermographic Signal Reconstruction (TSR) of Shepard et al. [5]. They fit a polynomial to the surface temperature evolution in double logarithmic scale. It allows the detection of defects based on the deviation of the polynomial from the linear decay. Additionally, noise reduction is achieved. A drawback is, that the coefficients of the fit have no physical interpretation. In contrast, we propose a decomposition of the temperature evolution into elementary solutions, where the coefficients of the fit are weights for temperature contributions from different depths and positions.

\section{Data Model}

We consider heating the structure under test by a very short thermal pulse, approximated by a Dirac pulse. The Dirac pulse is equivalent to a broad spectrum of thermal waves, which are all in phase [6]. For an object with a given thickness $Z_{\max }$, diffuse reflection and transmission occur at the interface between the rear surface and the surroundings. For common situations in pulse thermography, like the interface between metal and air, the reflection coefficient is close to one, thus transmission can be neglected. At the front surface, the reflected thermal waves lead to thermal contrasts between sound and defective areas, which is the basis for quantitative reconstruction algorithms, e.g., the Echo Defect Shape [7] or the Envelope Algorithm [8].

As a quantitative model for the thermal contrast at a certain surface point we consider the superposition of unilaterally propagated thermal waves according to Figure 1 . The contributions differ in intensity and in the length of the path the pulse has traveled before reaching the surface point under consideration.

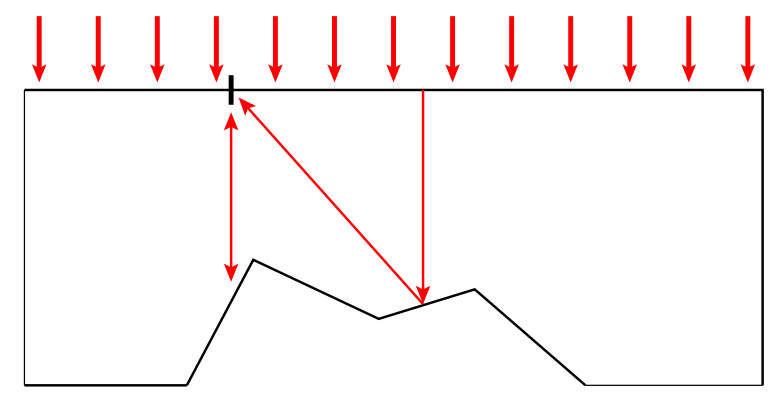

Fig. 1. The temperature evolution on the surface can be modeled as a superposition of contributions with different path lengths. 
The total surface temperature evolution then consists of the incoming heat pulse $\hat{T}$ as it would propagate through a semi-infinite specimen and the contributions of reflected thermal waves. As we consider only unilateral propagation, 1D heat transport models are employed. The incoming pulse $\hat{T}$ is described by the heat equation on $\Omega=[0, \infty)$ and the time interval $\left(0, t_{f}\right)$ :

$$
\begin{aligned}
\hat{T}_{t}(z, t)-\alpha \hat{T}_{z z}(z, t) & =0 & & \text { in } \Omega \times\left(0, t_{f}\right) \\
-\lambda \hat{T}_{z}(0, t)+h \hat{T}(0, t) & =h T_{\mathrm{ext}}+Q(t) & & \text { for } t \in\left(0, t_{f}\right) \\
\hat{T}(z, 0) & =T_{0} & & \text { for } z \in \Omega,
\end{aligned}
$$

where $\alpha=\frac{\lambda}{\rho c}$ with given material parameters density $\rho$, specific heat capacity $c$ and thermal conductivity $\lambda$. For the boundary condition, the heat transfer coefficient $h$ includes heat conduction and linearized radiation. The heating term is given by

$$
Q(t)= \begin{cases}q & t \leq t_{h} \\ 0 & t>t_{h}\end{cases}
$$

for the heating time $t_{h}$, describing flash or step heating used in practical applications. For $t_{h} \rightarrow 0, Q(t)$ could be idealized as a Dirac distribution.

Secondly we need to model the influence of the rear surface in depth $z_{f}$ at which the incoming pulse is reflected. This is essentially a prescribed heat flux at the rear. For a distance $L$ to the surface point under considerations, we obtain the following equation on $\Omega=[0, L]$ :

$$
\begin{aligned}
T_{t}(z, t)-\alpha T_{z z}(z, t) & =0 & & \text { in } \Omega \times\left(0, t_{f}\right) \\
-\lambda T_{z}(0, t)+h T(0, t) & =0 & & \text { for } t \in\left(0, t_{f}\right) \\
\lambda T_{z}(L, t) & =\tilde{Q}(t) & & \text { for } t \in\left(0, t_{f}\right) \\
T(z, 0) & =0 & & \text { for } z \in \Omega .
\end{aligned}
$$

Homogeneous boundary conditions are prescribed, as initial and external temperature are already included in Eq. 1). The heat flux $\tilde{Q}(t)=h\left(\hat{T}\left(z_{f}, t\right)-T_{0}\right)$ is determined by the solution of the semi-infinite problem. As can be easily verified, the surface contributions $T(0, t)$ depend only on the total path length $I=z_{f}+L$, such that they can be parametrized over just one parameter $l$.

\section{Reconstruction}

In order to reconstruct the surface temperature evolution at a certain point $x$ on the front surface of the specimen from noisy measurements, we assume the actual temperature $y(x, t)$ is a superposition of the incoming thermal pulse and the nonnegative contributions of reflected thermal waves $T_{l}(x, t)$ with different path lengths $/$ or travel times:

$$
y(x, t)=\hat{\theta} \hat{T}+\int_{l=0}^{l \max } \Theta(I) T_{l}(x, t) d l
$$

For a lateral size of $x_{\max }$ of the specimen and a thickness of $Z_{\max }$, the longest possible path length is given by $I_{\max }=$ $z_{\max }+\sqrt{z_{\max }^{2}+x_{\max }^{2}}$. However, as a contribution from the rear point directly below $x$ has a path length of at most $z_{\max }$, it will dominate all reflections arriving later. In practice, choosing $I_{\max }=2 z_{\max }$ is therefore sufficient. In the following, we assume the spatial position $x$ to be fixed.

As the area of the defect surface that leads to a specific path length / can vary, the intensity of the contributions and hence the coefficients $\theta(I) \geq 0$ of the superposition are unknown and have to be determined from the measured data $T_{\text {meas }}(x, t)$ :

$$
\min _{\theta \in L^{1}\left(0, l_{\text {max }}\right)}\left\|T_{\text {meas }}-\hat{\theta} \hat{T}-\int_{l=0}^{l_{\max }} \theta(I) T_{l} d l\right\|_{L^{2}\left(0, t_{f}\right)}^{2} \quad \text { subject to } \theta \geq 0
$$

Note that the non-negativity of $\theta$ and $T_{l}$ implies the stability of the reconstruction without further regularization. As regularization terms are strongly related to prior probability distributions (see [9]) of defect shapes, which are notoriously hard to formulate quantitatively, this is a major advantage of the present approach. The resulting temperature evolutions are necessarily smooth.

For the actual computation the path lengths have to be discretized. We employ piecewise constant functions and approximate the integral by the midpoint rule. As the measured data is obtained in form of a piecewise constant function in time, we approximate the $L^{2}$-norm by the midpoint rule with $n_{t}$ time points $t_{i}$ as well. Thus we end up with an inequality constrained linear least squares problem to be solved for each point $x$ of the front surface:

$$
\min _{\theta \in \mathbb{R}^{n_{I}}}\left\|T_{\text {meas }}-\hat{\theta} \hat{T}-T \theta\right\|^{2} \quad \text { subject to } \quad \theta \geq 0
$$


Therein, the matrix $T \in \mathbb{R}^{n_{t} \times n_{l}}$ given by $T_{i j}=T_{l_{j}}\left(x, t_{i}\right)$.

The least squares problem (4) can be solved independently for each pixel with trust-region methods [10] or active set methods [11] which are available, e.g., in the Matlab Optimization Toolbox.

\section{Green's Function Solutions}

For the set-up of the matrix $T$ in Eq. (4), the solutions $\hat{T}$ of 1 and $T_{l}$ of (2) have to be evaluated. In the following we derive analytical solutions.

\subsection{Semi-infinite domain}

by

We are seeking a Green's function solution to the heat equation 11. The Green's function for this problem is given

$$
\begin{gathered}
G(z, t ; \xi, \tau)=\frac{1}{2 \sqrt{\pi \alpha(t-\tau)}}\left(\exp \left(-\frac{(z-\xi)^{2}}{4 \alpha(t-\tau)}\right)+\exp \left(-\frac{(z+\xi)^{2}}{4 \alpha(t-\tau)}\right)\right) \\
-\frac{h}{\lambda} \exp \left(\frac{h^{2}}{\lambda^{2}} \alpha(t-\tau)+\frac{h}{\lambda}(z+\xi)\right) \operatorname{erfc}\left(\frac{z+\xi}{2 \sqrt{\alpha(t-\tau)}}+\frac{h}{\lambda} \sqrt{\alpha(t-\tau)}\right)
\end{gathered}
$$

see e.g. [12]. Thus the solution to Eq. 11 can be computed as

$$
\hat{T}(z, t)=\int_{0}^{\infty} T_{0} G(z, t ; \xi, 0) d \xi+\frac{\alpha}{\lambda} \int_{0}^{t} Q(\tau) G(z, t ; 0, \tau) d \tau+\frac{\alpha}{\lambda} \int_{0}^{t} h T_{\text {ext }} G(z, t ; 0, \tau) d \tau .
$$

Each integral can be evaluated exactly, see appendix $\mathrm{A}$

\subsection{Reflection contributions}

A Green's function for problem (2) is given by

$$
G(z, t ; \xi, \tau)=\frac{2}{L} \sum_{m=1}^{\infty} \exp \left(-\frac{\beta_{m}^{2} \alpha(t-\tau)}{L^{2}}\right)\left(\frac{\beta_{m}^{2}+B^{2}}{\beta_{m}^{2}+B^{2}+B}\right) \cos \left(\beta_{m}\left(1-\frac{z}{L}\right)\right) \cos \left(\beta_{m}\left(1-\frac{\xi}{L}\right)\right) .
$$

Here, $B=L \frac{h}{\lambda}$ and $\beta_{m}$ are the solutions to $\beta_{m} \tan \beta_{m}=B$ in ascending order.

The surface temperature $T_{L}(0, t)$ can be computed as

$$
\begin{aligned}
T_{L}(0, t) & =\frac{\alpha}{\lambda} \int_{0}^{t} \tilde{Q}(\tau) G(0, t ; L, \tau) d \tau \\
& =\frac{2 \alpha}{\lambda L} \sum_{m=1}^{\infty}\left(\frac{\beta_{m}^{2}+B^{2}}{\beta_{m}^{2}+B^{2}+B}\right) \cos \left(\beta_{m}\right) \int_{0}^{t} \tilde{Q}(\tau) \exp \left(-\frac{\beta_{m}^{2} \alpha(t-\tau)}{L^{2}}\right) d \tau .
\end{aligned}
$$

The heat flux $\tilde{Q}(t)$ is determined by the solution of the semi-infinite problem, $\tilde{Q}(t)=h\left(\hat{T}(L, t)-T_{0}\right)$. Note that we do not need to include the reflection coefficient, as it is implicitly determined through the solution of the least squares problem (4).

The integral is evaluated using numerical quadrature, the infinite sum is approximated by summing to a suitable cut-off parameter $M$. The resulting temperature evolutions are illustrated in Figure 2.

\section{Results}

To check the proposed algorithm, PVC test specimens with triangular wedge-shaped defects were used for generating simulation data and for measurements, see Figure 3 for a sketch. As the defect is uniform in $y$-direction, in the following we choose an arbitrary cross section for the defect shape reconstruction. 

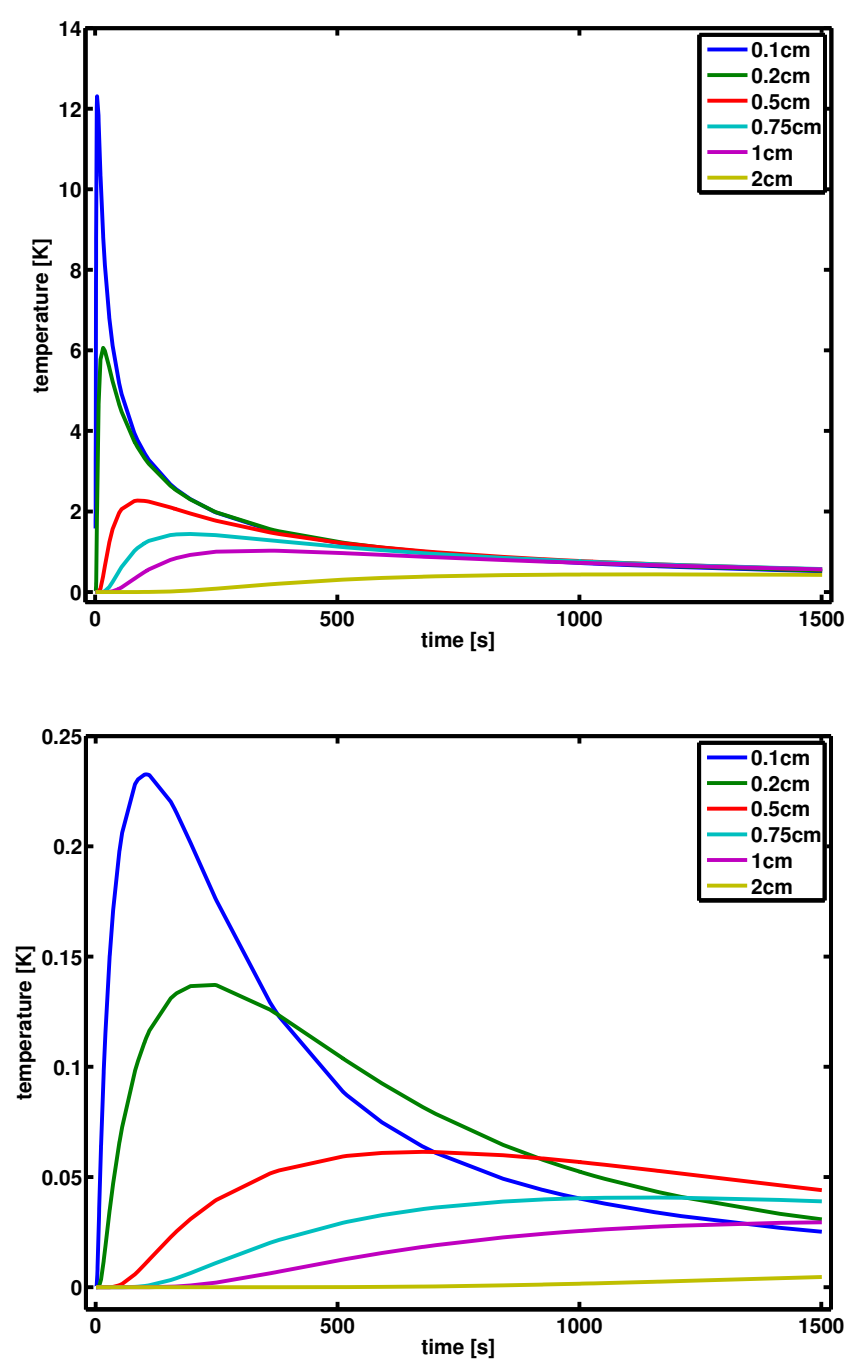

Fig. 2. Top: Solutions $\hat{T}\left(z_{i}, t\right)$ to Eq. 1 for $T_{0}=T_{\text {ext }}=0$ and different depths $z_{i}$. Bottom: Front surface temperature $T_{L_{i}}(0, t)$ for Eq. 2 for rear in different depths $L_{i}$.

\subsection{Defect shape reconstruction}

As the overall aim is to extract quantitative information about defect size and shape, we are not only interested in the quality of the fit, but also in the influence of the proposed preprocessing on defect shape identification algorithms. To reconstruct the defect, we use the Envelope Algorithm [8], a variant of the Echo Defect Shape method [7]. In the latter, the relative thermal contrast

$$
C_{\text {rel }}(t)=\frac{T_{\text {defect }}(t)-T_{\text {reference }}(t)}{T_{\text {reference }}(t)}
$$

is used to extract the thickness of the specimen at each surface point based on the travel time of the reflection of thermal pulse in a purely $1 \mathrm{D}$ approximation. This depth estimate can be iteratively improved using comparatively expensive numerical simulations of the heat equation. In the Envelope Algorithm, the thermal contrast $C_{\text {rel }}$ is used to construct parabolas of equal travel time, thus taking lateral heat conduction to some extent into account. The envelope of the parabolas is a good approximation of the defect shape, comparable in quality to the Echo Defect Shape with a couple of iterative refinement steps.

\subsection{Simulation data}

Simulation data was generated by the semi-analytic method [13], with material parameters $\lambda=0.16 \frac{\mathrm{W}}{\mathrm{mK}}, \rho=$ $1500 \frac{\mathrm{kg}}{\mathrm{m}^{3}}, c=900 \frac{\mathrm{J}}{\mathrm{kg} \mathrm{K}}, h=6.28 \frac{\mathrm{W}}{\mathrm{m}^{2} \mathrm{~K}}, C_{\text {trans }}=6.67051 \cdot 10^{-8} \frac{\mathrm{W}}{\mathrm{m}^{2} \mathrm{~K}^{4}}$. The initial and external temperature was $T_{\text {ext }}=T_{0}=$ 

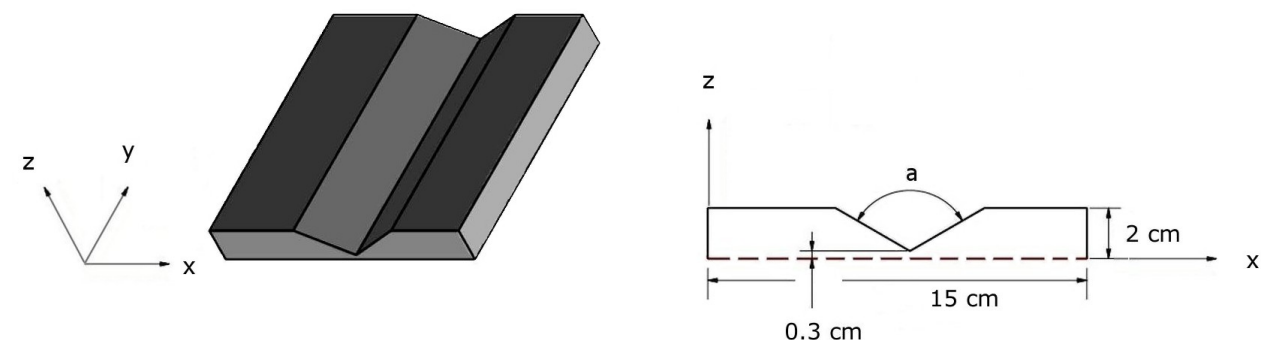

Fig. 3. PVC test specimen with wedge-shaped defect. The angle a determines the defect shape and size.

$T_{\mathrm{amb}}=298 \mathrm{~K}$. We incorporated a radiation boundary condition linearized at $T_{0}$, i.e. used $\tilde{h}=h+4 c_{\text {trans }} T_{0}^{3}$ in the boundary conditions $1 \mathrm{~b}, 2 \mathrm{2b}$.

We added Gaussian white noise with standard deviation $\sigma_{1}=0.2 \mathrm{~K}$ and $\sigma_{2}=1 \mathrm{~K}$. Fitting results can be seen in Figure 4 for the $a=120^{\circ}$-wedge. Although the temperature evolution is fitted for each surface pixel separately, a clear improvement of the data in a spatial cross section (at a fixed time) can be observed. Despite the high noise level, the temperature rise above the defect is clearly visible.

For applying the Envelope Algorithm [8], we need additional smoothing in space. For simplicity, we used a standard Gauss filter in space on the noisy data after applying the Green decomposition for smoothing in time. We compare this to the results for the same Gauss filter in space combined with a moving average filter in time. Instead of using reference data from a defect-free specimen, we used the solution $\hat{T}(0, t)$ as a reference for computing the relative thermal contrast $C_{\text {rel }}$ used in the reconstruction algorithm.

The reconstruction results can be seen in Figure 5 . While the reconstruction for smoothing by the moving average filter neither captures the top of the defect, nor the flanks and lateral extent, both are resolved quite well using the Green fit.

\subsection{Experimental data}

For the measurements, the experimentally determined material parameters of the PVC test specimens were thermal conductivity $\lambda=0.213 \pm 0.02 \frac{\mathrm{W}}{\mathrm{mK}}$, density $\rho=1413.4 \pm 4 \frac{\mathrm{kg}}{\mathrm{m}^{3}}$ and specific heat capacity $c=1038 \pm 40 \frac{\mathrm{J}}{\mathrm{kgK}}$. The heating was performed using two flash lights with $6 \mathrm{~kJ}$ emission energy and a flash duration of $3 \mathrm{~ms}$. The cooling curve was recorded with a Thermosensorik InSb 640 IR-camera. The recording frequency was originally $93 \mathrm{~Hz}$, which was reduced to $1 \mathrm{~Hz}$ by in-camera averaging. Figure 6 shows the surface temperature directly after flash heating, as well as after $22 s$ of the cooling phase. Inhomogeneous heating, as well as measurement noise are clearly visible.

After temporal smoothing, a method for spatial smoothing has to be applied. We combined the moving average filter with Gaussian smoothing (the standard tools from image enhancement). TSR and the present approach were combined with a Bayesian type smoothing, where a priori information about the temperature evolution in a defect free sample is used to construct an a posteriori estimate of the data using the Bayes theorem. See Appendix $B$ for a brief sketch of the method, and, e.g., [9, 14] for an general introduction to the Bayesian approach to the solution of inverse problems. According to our experience, Bayesian spatial smoothing gives slightly better results than a standard Gauss filter.

As a reference temperature for contrast computation, the average of a small defect-free patch of the test specimen was used. Figures 7 and 8 show the resulting fitted temperatures and reconstructed defect shapes. As for the simulation data, the top and flanks of the defect are resolved best using the Green decomposition method.

\section{Conclusion}

We have presented an algorithm for enhancement of active thermography data that is based on Green's functions for idealized 1D heat conduction problems. A major advantage is that the coefficients are known to be nonnegative for physical reasons, such that further, often quite arbitrary, regularization is not required to obtain smooth temperature reconstructions. Due to analytically available ansatz functions and the spatially decoupled computation of coefficients, the method is efficient and easy to implement, though somewhat more complex than moving averages or polynomial fitting.

Compared to simpler methods for data enhancement, the resulting defect reconstructions appear to be consistently better for both simulation data and measurements. Nevertheless, additional spatial smoothing is still required for good reconstruction results. 

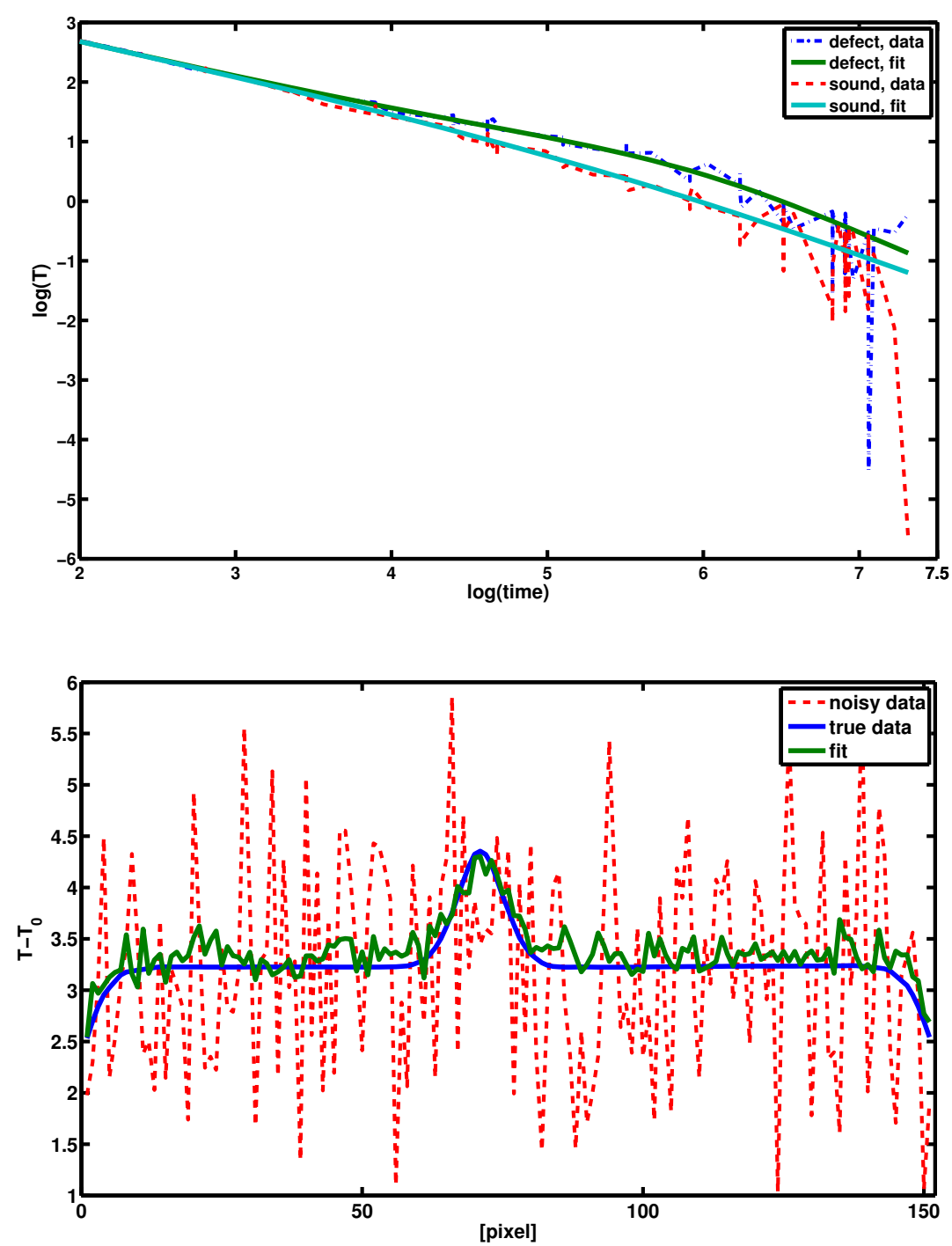

Fig. 4. Simulation data. Top: Fitted surface temperature above defect and sound area for the $a=120^{\circ}$-triangle and $\sigma_{1}=0.2 \mathrm{~K}$ (detail). Bottom: Temperature over a cross section in space at $t=87.5 \mathrm{~s}$ for $\sigma_{2}=1 \mathrm{~K}$.

\section{A. Computation of $\hat{T}$}

The solution to Eq. 1] can be computed as

$$
\hat{T}(z, t)=\underbrace{\int_{0}^{\infty} T_{0} G(z, t ; \xi, 0) d \xi}_{=\hat{T}^{\mathrm{ic}}(z, t)}+\underbrace{\frac{\alpha}{\lambda} \int_{0}^{t} Q(\tau) G(z, t ; 0, \tau) d \tau}_{=\hat{T}^{\mathrm{ht}}(z, t)} \underbrace{+\frac{\alpha}{\lambda} \int_{0}^{t} h T_{\mathrm{ext}} G(z, t ; 0, \tau) d \tau}_{=\hat{T}^{\mathrm{bc}}(z, t)} .
$$

Each integral can be evaluated exactly:

$$
\begin{gathered}
\hat{T}^{\mathrm{bc}}(z, t)=T_{\text {ext }}\left[\operatorname{erfc}\left(\frac{z}{2 \sqrt{\alpha t}}\right)-\exp \left(\frac{h^{2}}{\lambda^{2}} \alpha t+\frac{h}{\lambda} z\right) \operatorname{erfc}\left(\frac{z}{2 \sqrt{\alpha t}}+\frac{h}{\lambda} \sqrt{\alpha t}\right)\right] \\
\hat{T}^{\mathrm{ic}}(z, t)=T_{0}\left[1-\operatorname{erfc}\left(\frac{z}{2 \sqrt{\alpha t}}\right)+\exp \left(\frac{h^{2}}{\lambda^{2}} \alpha t+\frac{h}{\lambda} z\right) \operatorname{erfc}\left(\frac{z}{2 \sqrt{\alpha t}}+\frac{h}{\lambda} \sqrt{\alpha t}\right)\right]
\end{gathered}
$$

For $T_{\text {ext }}=T_{0}$, we have $\hat{T}^{\mathrm{ic}}(z, t)+\hat{T}^{\mathrm{bc}}(z, t)=T_{0}$. In this case, only the heating term needs to be computed. There, we need to distinguish between $t \leq t_{h}$ and $t>t_{h}$. For $t \leq t_{h}$, 

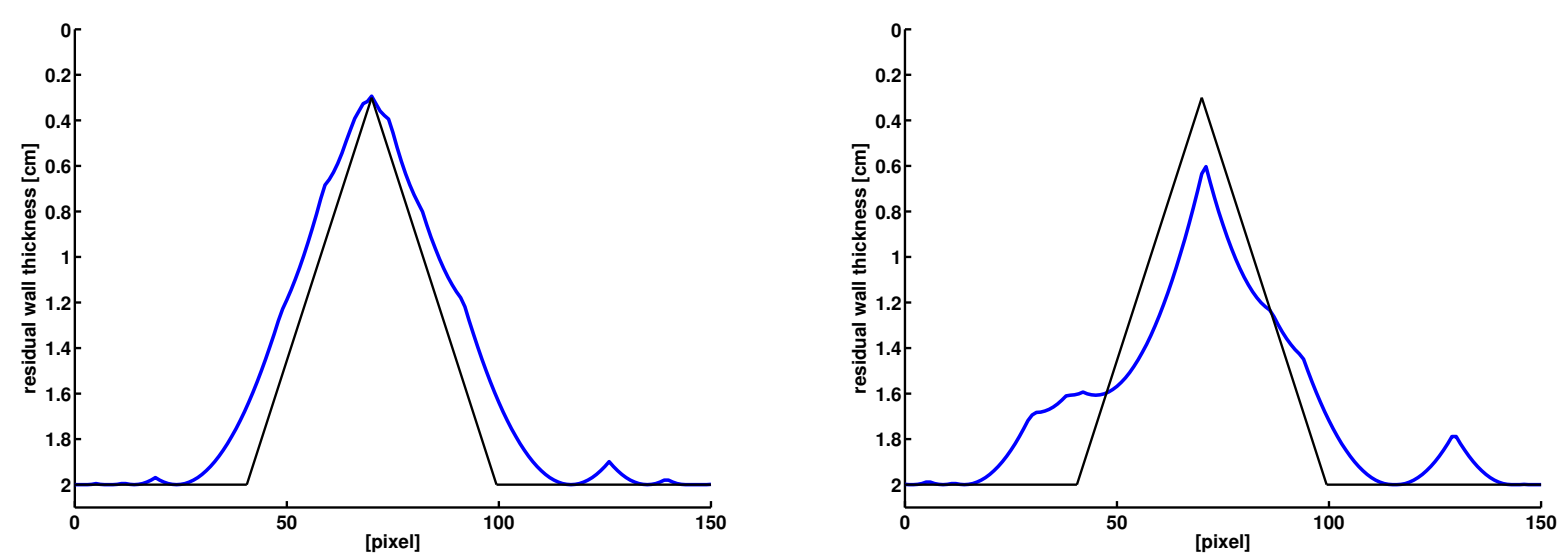

Fig. 5. Simulation data. Reconstruction using the Envelope Algorithm [8] for the $a=120^{\circ}$-triangle and $\sigma_{2}=1 \mathrm{~K}$ with Green (left) and moving average (right), both applied before using Gauss filter in space with $\sigma^{2}=0.5$. As a reference temperature for contrast computation, $\hat{T}(0, t)$ was used. Thresholds for the thermal contrast were 0.075.
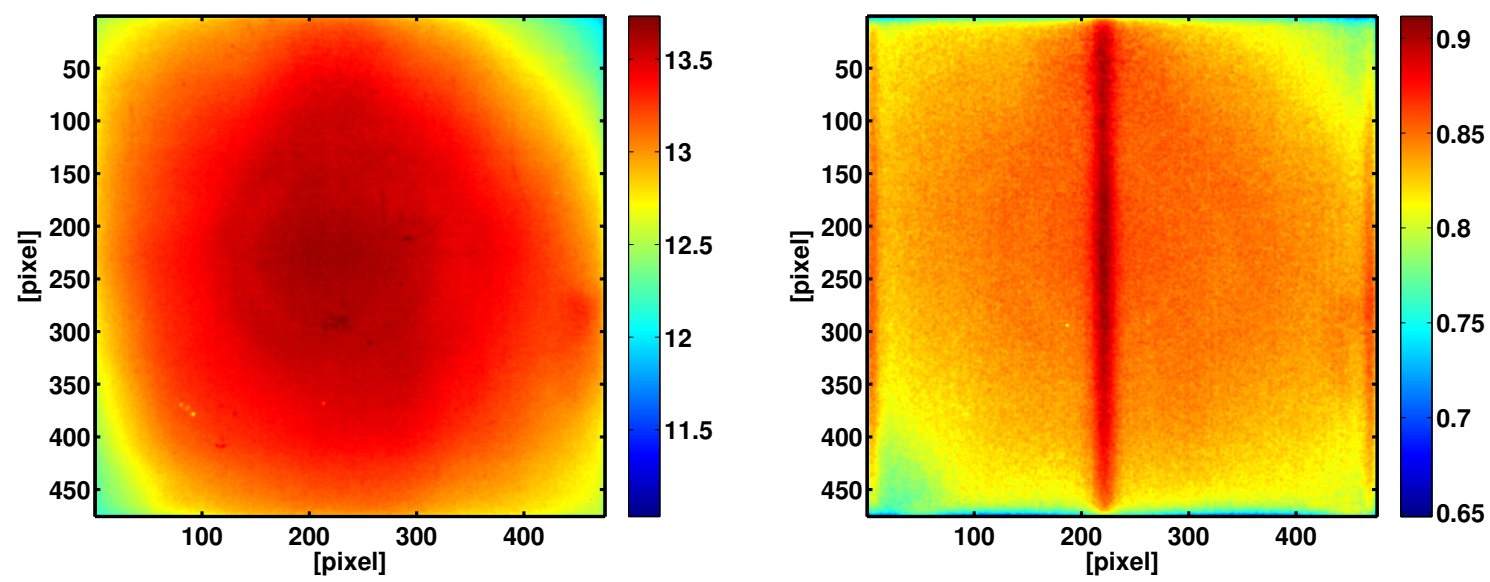

Fig. 6. Experimental data: Surface temperature directly (left) and 22s (right) after heating.

$$
\hat{T}^{\mathrm{ht}}(z, t)=\frac{q}{h}\left[\operatorname{erfc}\left(\frac{z}{2 \sqrt{\alpha t}}\right)-\exp \left(\frac{h^{2}}{\lambda^{2}} \alpha t+\frac{h}{\lambda} z\right) \operatorname{erfc}\left(\frac{z}{2 \sqrt{\alpha t}}+\frac{h}{\lambda} \sqrt{\alpha t}\right)\right]
$$

holds. For $t>t_{h}$, we obtain

$$
\begin{aligned}
\hat{T}^{\text {ht }}(z, t) & =\frac{q}{h}\left[\operatorname{erfc}\left(\frac{z}{2 \sqrt{\alpha t}}\right)-\exp \left(\frac{h^{2}}{\lambda^{2}} \alpha t+\frac{h}{\lambda} z\right) \operatorname{erfc}\left(\frac{z}{2 \sqrt{\alpha t}}+\frac{h}{\lambda} \sqrt{\alpha t}\right)\right] \\
& +\frac{q}{h}\left[\exp \left(\frac{h^{2}}{\lambda^{2}} \alpha\left(t-t_{h}\right)+\frac{h}{\lambda} z\right) \operatorname{erfc}\left(\frac{z}{2 \sqrt{\alpha\left(t-t_{h}\right)}}+\frac{h}{\lambda} \sqrt{\alpha\left(t-t_{h}\right)}\right)-\operatorname{erfc}\left(\frac{z}{2 \sqrt{\alpha\left(t-t_{h}\right)}}\right)\right] .
\end{aligned}
$$

\section{B. Bayesian data smoothing}

In this section, we briefly sketch the Bayesian approach used for spatial data smoothing.

The basic idea of the Bayesian approach to the solution of inverse problems is to remove the ill-posedness by restating the problem in a larger space of probability distributions. All variables are modeled as random variables and information about the variables is coded in probability distributions [9]. 


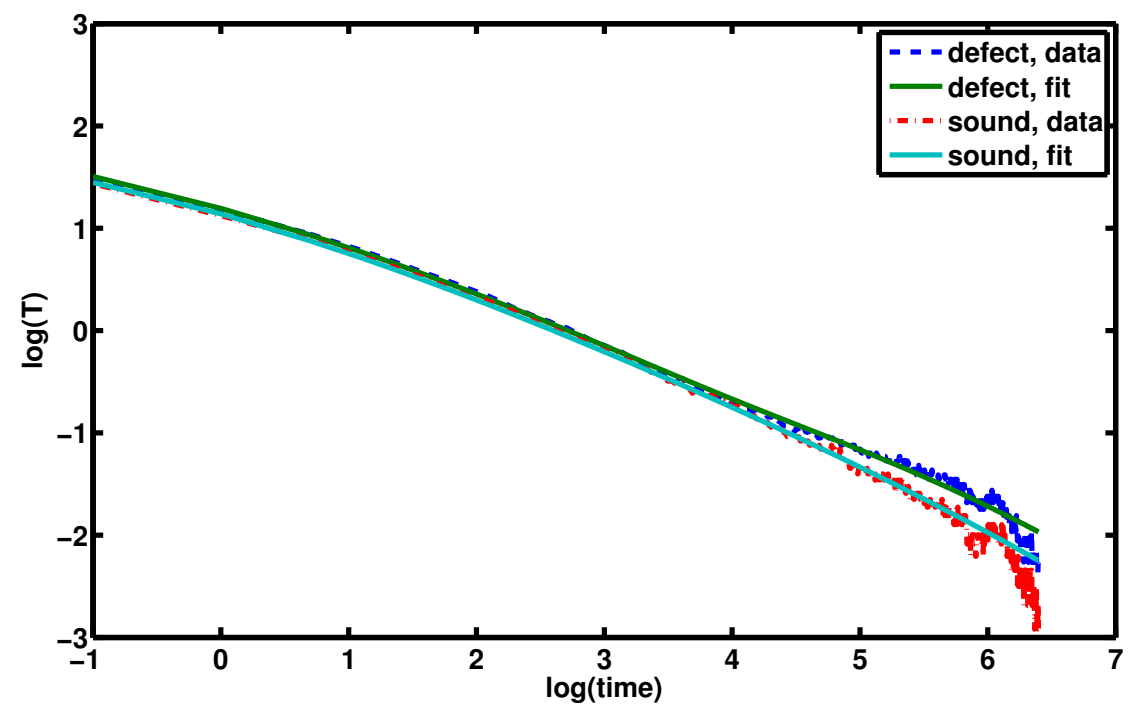

Fig. 7. Experimental data: Fitted surface temperature above defect and sound area (detail).

Here, we use this technique to approximate the true temperature of the specimen from the measured data. Let $T$ denote the true temperature and $y$ the measurement. Then the theorem of Bayes states, that $p(T \mid y) \propto p(y \mid T) p(T)$. The likelihood distribution $p(y \mid T) \propto \exp \left(-\frac{1}{2 \sigma^{2}}\|y-T\|\right)$ encodes the statistics of measurement errors, here modeled as additive white noise. the prior distribution $p(T)$ encodes the statistics of the true temperatures. As this distribution is rarely well-known, a reasonable modeling of the prior is one of the most difficult tasks in Bayesian inversion. Here, for lack of more precise knowledge, we choose the normal distribution $p(T) \propto \exp \left(-\frac{1}{2}(\|T-\tilde{T}\|+\|L x\|)\right)$, where $L$ is a discretized Laplace operator (smoothness prior) and

$$
\tilde{T}(t)=\frac{q}{\sqrt{4 \pi \rho \lambda c t}}\left(1+\exp \left(-\frac{\rho c}{\lambda t} z_{\max }^{2}\right)\right)
$$

describes the approximate temperature evolution in a defect-free specimen. The result of Bayesian inversion is then the posterior distribution $p(T \mid y)$. As computation and interpretation of high-dimensional probability distributions is in general extremely difficult, usually only point-estimates are computed, e.g., the conditional mean $T_{\mathrm{CM}}=E(T \mid y)$ of the posterior distribution as used here. As all probability distributions are normal in the comparatively simple setting considered here, the resulting posterior is also a normal distribution, and we can compute the conditional mean explicitly. $T_{\mathrm{CM}}$ then is used as input for the defect shape reconstruction algorithm.

\section{REFERENCES}

[1] X. P. V. Maldague. Theory and practice of infrared technology for nondestructive testing. Wiley, New York, 2001.

[2] S. Rainieri and G. Pagliarini. Data filtering applied to infrared thermographic measurements intended for the estimation of local heat transfer coefficient. Experimental Thermal and Fluid Science, 26:109-114, 2002.

[3] Z. Alsaadawi and U. Netzelmann. Spatio-temporal filtering of active thermography data for noise reduction and data compression. In X. P. V. Maldague, editor, Proceedings of the 10th edition of the Quantitative Infrared Thermograpy International Conference, number QIRT2010-006, 2010.

[4] S. D. Holland and J. Renshaw. Physics-based image enhancement for infrared thermography. NDT\&E International, 43(5):440-445, 2010.

[5] S. M. Shepard, J. R. Lhota, B. A. Rubadeux, D. Wang, and T. Ahmed. Reconstruction and enhancement of active thermographic image sequences. Opt. Eng., 42(5):1337-1342, 2003.

[6] D. Almond and P. Patel. Photothermal science and techniques. Chapman \& Hall, London, 1996.

[7] S. Lugin and U. Netzelmann. A defect shape reconstruction algorithm for pulsed thermography. NDT\&E International, 40(2):220-228, 2007.

[8] S. Götschel, M. Weiser, Ch. Maierhofer, R. Richter, and M. Röllig. Fast defect shape reconstruction in travel time pulsed thermography. In O. Büyüköztürk, M. A. Taşdemir, O. Güneş, and Y. Akkaya, editors, Nondestructive Testing of Materials and Structures, 2012. 


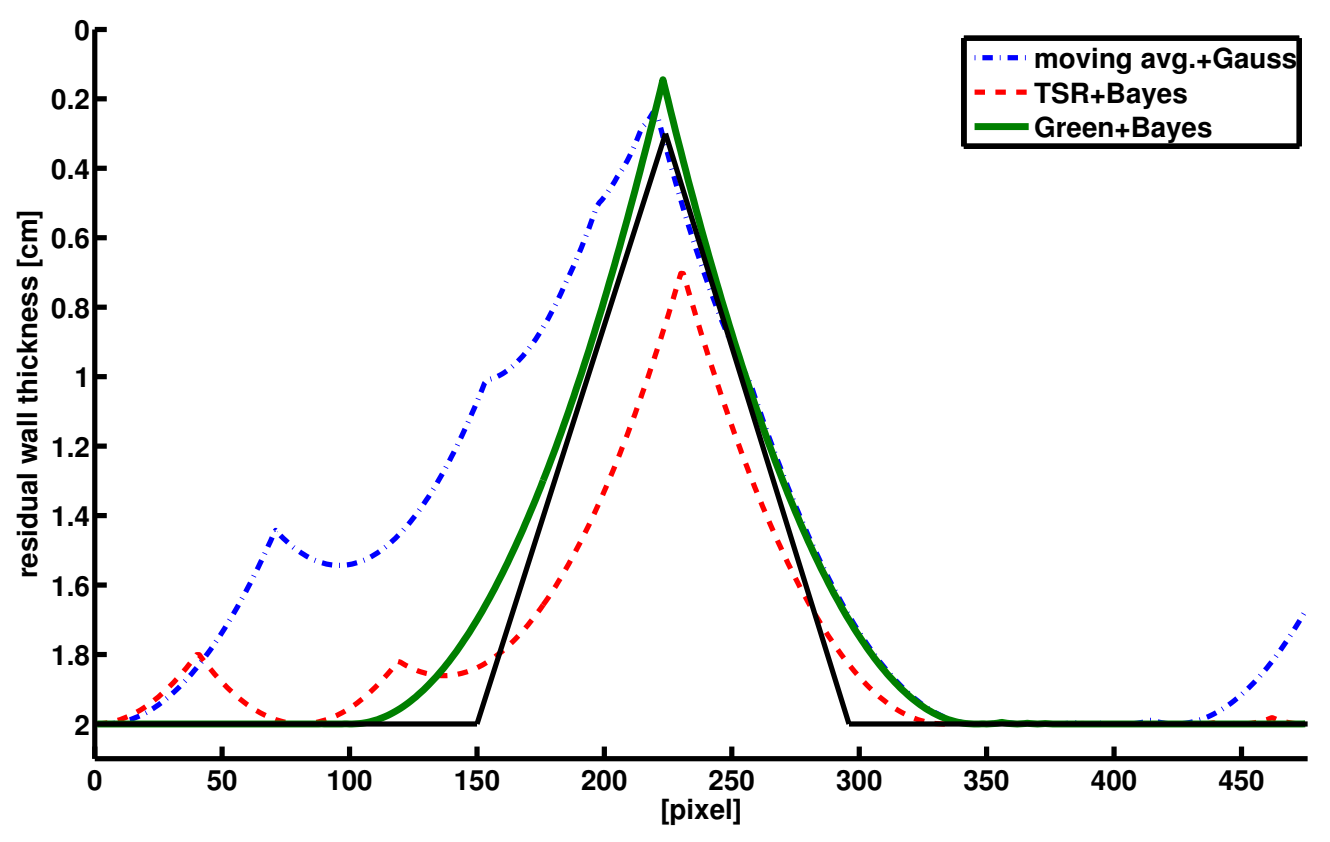

Fig. 8. Experimental data: Reconstruction for the $120^{\circ}$-triangle for different preprocessing techniques.

[9] J. Kaipio and E. Somersalo. Statistical and Computational Inverse Problems, volume 160 of Applied Mathematical Sciences. Springer, New York, 2005.

[10] T. F. Coleman and Y. Li. A reflective newton method for minimizing a quadratic function subject to bounds on some of the variables. SIAM Journal on Optimization, 6(4):1040-1058, 1996.

[11] P. E. Gill, W. Murray, and M. H. Wright. Practical Optimization. Academic Press, London, 1981.

[12] K. D. Cole, J. V. Beck, A. Haji-Sheikh, and B. Litkouhi. Heat Conduction Using Green's Functions. CRC/Taylor and Francis, Boca Raton, 2nd edition, 2011.

[13] M. Weiser, M. Röllig, R. Arndt, and B. Erdmann. Development and test of a numerical model for pulse thermography in civil engineering. Heat and Mass Transfer, 46(11):1419-1428, 2010.

[14] A. Stuart. Inverse problems: a Bayesian perspective. Acta Numerica, 19:451-559, 2010. 